\title{
Tuberculosis of the skin
}

\author{
B M G D Yasaratne ${ }^{1}$, D M Madegedara² \\ Journal of the Ceylon College of Physicians, 2010, 41, 83-88
}

\section{Introduction}

Though one of the oldest diseases known to affect mankind, tuberculosis (TB) still continues to be a significant health problem worldwide. Improved living standards, health education, effective screening and treatment facilities have greatly reduced the prevalence of TB in many industrialized countries. Nevertheless resurgence of infection is being witnessed in some areas especially with the increased use of immunosuppressive therapy, emergence of metabolic diseases and acquired immunodeficiency syndrome (AIDS) epidemic. In the developing world TB remains a major public health issue despite strenuous containment efforts ${ }^{1,2}$.

Extrapulmonary TB constitutes approximately $10 \%$ of all cases of TB and is on the rise due to compromised host immunity ${ }^{3}$. Infections of the skin due to mycobacterium tuberculosis form a bulk of the historic background of dermatology, where scrofuloderma, the King's evil and lupus vulgaris once ravaged faces across Europe and many parts of the world. Presently cutaneous TB is rare and makes up only 0.1 to $1.5 \%$ of all new cases worldwide, but in high prevalent settings can be up to 2.5 percent t $^{4,5,6,7}$.

We found two published case series of cutaneous tuberculosis reported from Sri Lanka. Atukorala et al presented a case series of fifteen patients of whom twelve had lupus vulgaris and three had scrofuloderma ${ }^{8}$. Vyravanathan et al reported five cases of scrofuloderma over the sternum ${ }^{9}$. De Siva et al reported a case of erythema induratum in a patient with active endometrial tuberculosis ${ }^{10}$.

A thorough understanding of different presentations of cutaneous TB is useful for the practicing physician in both adult and paediatric medicine, where they would be required to recognize the early disease at outpatient clinics. In this brief review, we discuss the different manifestations, subsequent work-up, treatment aspects and other concerns of practical significance with reference to cutaneous TB in the high prevalence local setting.

\footnotetext{
${ }^{1}$ Senior Registrar in Respiratory Medicine,

${ }^{2}$ Consultant Respiratory Physician, Respiratory Research Unit, Teaching Hospital, Kandy.
}

\section{Classification and clinical variants}

The most widely accepted classification system for cutaneous TB is based on the mechanism of propagation. The concept of bacterial load has been added to the classification (Table 1), where in multibacillary forms, direct visualization of Ziehl-Nielson stained organism from skin biopsy is readily possible ${ }^{11,12,13,14}$. Tuberculids are symmetric generalized exanthems in the skin of patients, possibly resulting from hypersensitivity reactions to tubercle bacillus.

\section{Multibacillary forms}

\section{Primary-inoculation TB (tuberculous chancre)}

Primary-inoculation TB results from direct introduction of mycobacteria into the skin or mucosa of an individual who was not previously infected with TB. It is commonly seen in health care or laboratory worker who has acquired TB through accidental inoculation of contaminated material or in children with positive contact who are not immunized with the $M$ bovis strain bacille Calmette-Guérin (BCG).

Common sites for primary inoculation TB include the face and other exposed skin, but also can be seen with ritual circumcision, tattooing, ear piercing, etc. Acute primary gingivitis can occur after ingestion of milk contaminated with $M$ bovis. The skin lesion will start as a papule or a nodule that will eventually ulcerate in 2 to 3 weeks to form a firm, nonhealing, shallow, nontender, undermined ulcer with a granulomatous base. Painless regional lymphadenopathy is evident at 3-8 weeks producing a clinical picture of a lymphocutaneous complex analogous to the Ghon complex (Figure 1). Lesions may resolve spontaneously or progress into a lupus vulgaris like picture and rarely, primaryinoculation TB may lead to disseminated disease.

\section{Scrofuloderma}

Scrofuloderma is the result of continuous propagation of infection to involve the skin from an underlying structure, most commonly a lymph node, bone or joint and is the most common form of cutaneous TB in many series. Primarily affected areas are the neck, axillae, chest wall and groin. Commonly, an oral or tonsillar primary lesion following contaminated milk ingestion progresses to cervical adenitis, which eventually leads to a cold abscess with fistula formation and secondary breakdown of overlying cervical skin. 
Lesions present as firm, painless, subcutaneous nodules that gradually enlarge and suppurate and then form ulcers and sinus tracts in overlying skin (Figure 2). The differential diagnoses include actynomicosis, hydradenitis supurativa, granuloma inguinale and lymphogranuloma venerum. Spontaneous healing can occur but often takes years and is accompanied by the formation of keloid tissue or hypertrophic scars. Lupus vulgaris may develop in the vicinity of healing scrofuloderma while haematogenous spread may lead to tuberculous gumma or active pulmonary or pleural disease with systemic symptoms.

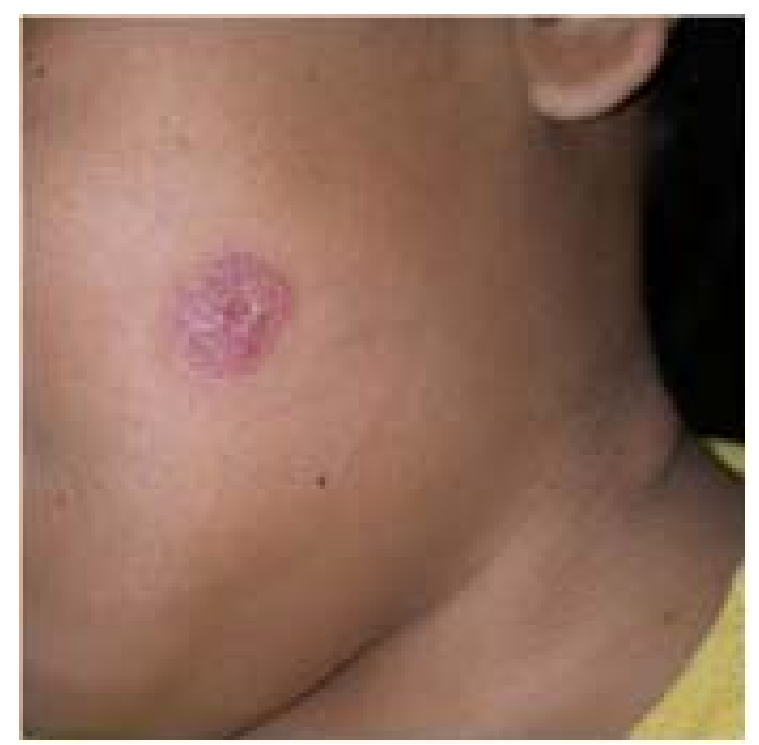

Figure 1. Primary inoculation TB of the exposed skin with painless regional lymphadenopathy.

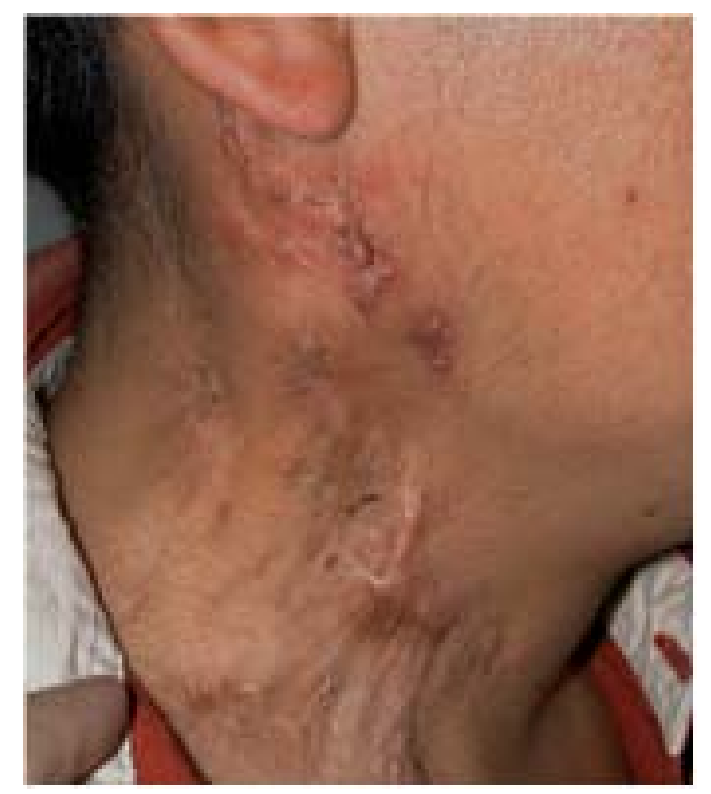

Figure 2. Suppurative ulcers and sinus tracts of scrofuloderma.

\section{Tuberculosis periorificialis}

Periorificial TB results from autoinoculation of mycobacteria into the periorificial skin and mucous membranes in patients with advanced TB. In perioral TB primary site is usually upper airways or lungs, while perineal TB is secondary to intestinal or genitourinary disease. The most common lesion is a painful ulcer or a plaque with a pseudomembranous fibrinous base, which should be differentiated from parasitic or fungal disease and malignancy. This form of cutaneous TB is rare and usually affects older men. Tuberculin sensitivity is strong. Tubercles with acid-fast bacilli can be found in the deep dermis and ulcer walls. Prognosis is usually poor due to severe internal organ disease.

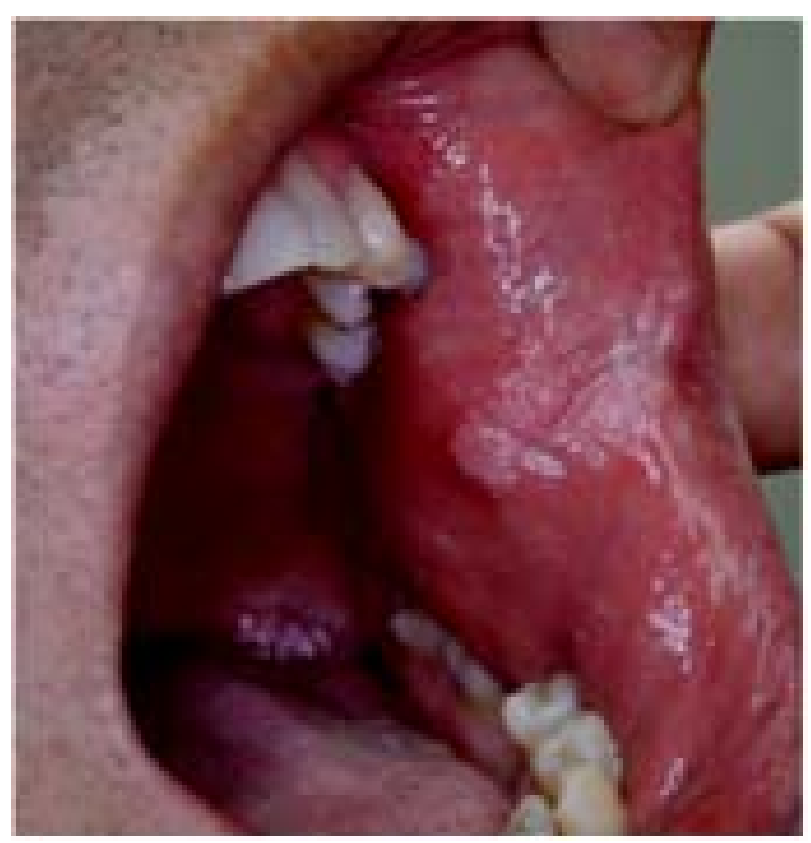

Figure 3. Periorificial TB presenting as a painful ulcer with indurations.

\section{Acute miliary tuberculosis}

Acute miliary TB variant is usually seen in children and adolescents with advanced pulmonary or disseminated TB. The trunk is the most common location, where small erythematous macules or papules develop that become necrotic later. Histology will demonstrate necrotizing tuberculous granuloma with multiple acid-fast bacilli, although the tuberculin test may be negative. 


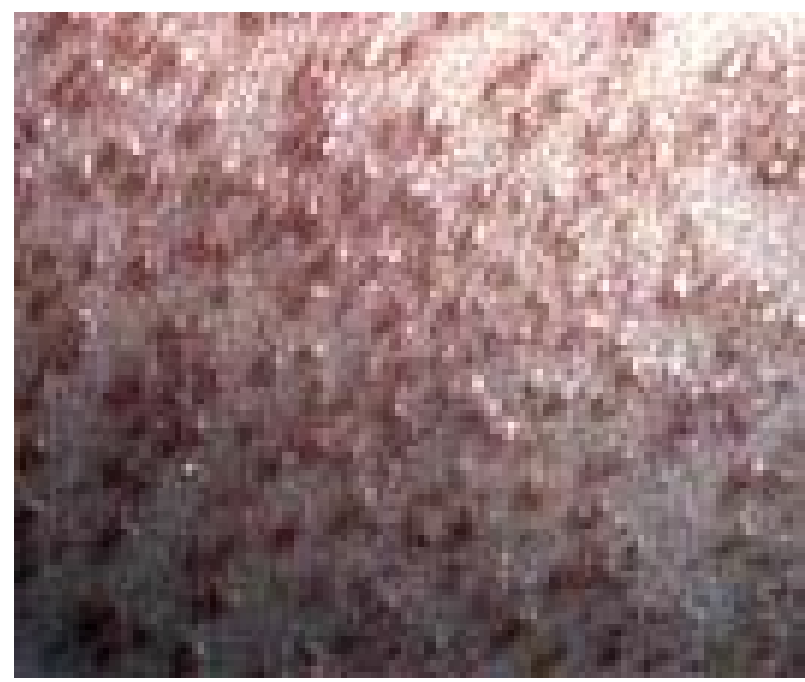

Figure 4. Erythematous papules of acute miliary TB of skin.

\section{Tuberculous gumma}

Gummas are cold abscesses that will develop at extremities or on the trunk as a result of haematogenous spread from dormant mycobacteria in patients without underlying disease. Histology shows suppurative granulomata with nonspecific infiltrates which will usually demonstrate the presence of the mycobacteria. The tuberculin test is usually positive but may be negative if associated with poor general condition.

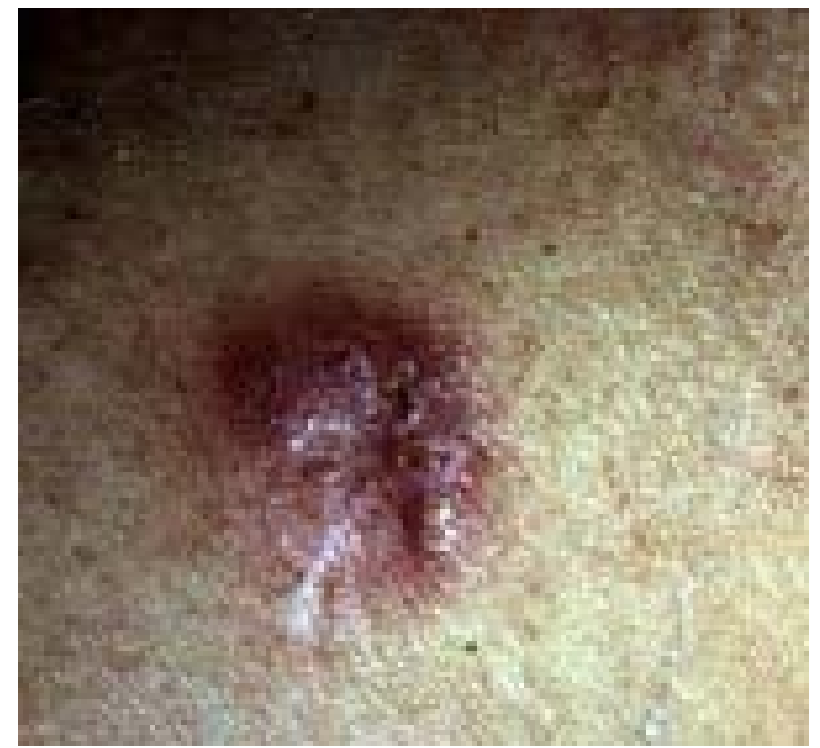

Figure 5. TB gumma of an extremity.

\section{Paucibacillary forms}

\section{Tuberculosis verrucosa cutis}

Verrucous TB results as a consequence of reinoculation of mycobacteria in an individual with previous exposure and is characterized by the presence of a solitary, verrucose plaque, usually on an extremity such as the hand or the foot. Infection starts as an asymptomatic warty papule with a slow growth and irregular peripheral extension. The lesion may show central involution with an atrophic scar or form massive papillary excrescence with fissures. Lesions usually are painless and solitary, and regional nodes are generally not affected.

Reinfection can result from accidental exposure to tuberculous tissue in high-risk groups, such as physicians, pathologists, laboratory workers, veterinarians, farmers and butchers. Histology may reveal poorly defined noncaseating, tuberculous granulomata. The tuberculin test is strongly positive, but mycobacteria are generally not isolated from lesions. The lesion should be differentiated from verrucous warts and verrucous carcinoma of skin.

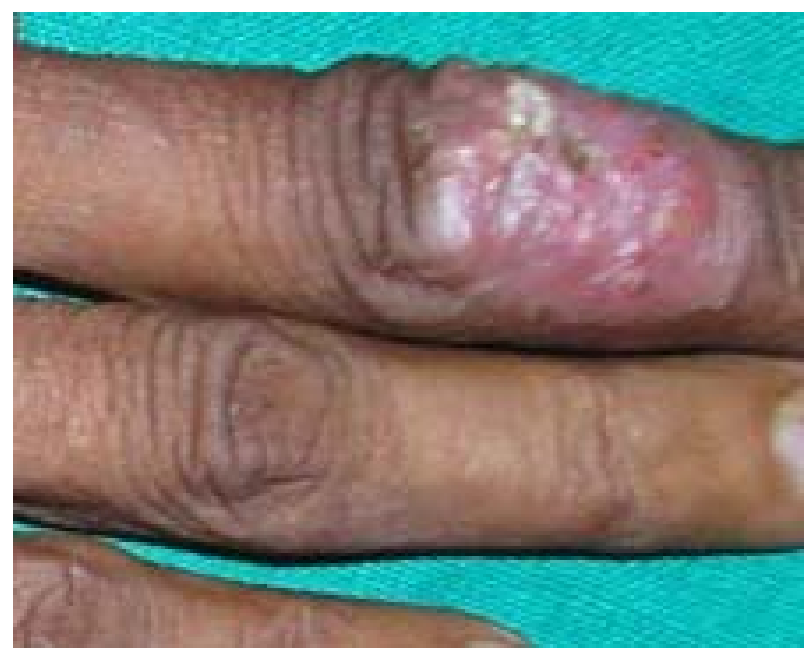

Figure 6. TB verrucosa cutis on a finger.

\section{Lupus vulgaris}

This is the most common form of cutaneous TB in many parts of the world with a female preponderance of 2-3:1. Facial lesions usually follow haematogenous spread, while direct inoculation is responsible for many lesions at extrimities. Lupus vulgaris may also follow direct extension or lymphatic spread from underlying tuberculous foci, BCG vaccination or scrofuloderma.

Head and neck is involved in more than $90 \%$ of cases. Characteristically lesions are solitary, small, sharply marginated, red-brown papules of gelatinous consistency (apple-jelly nodules), which slowly evolve by peripheral extension and central atrophy into large plaques. Other than the plaque form, ulcerative, vegetative and nodular forms of lupus vulgaris have been described. 
The histology will show multiple noncaseating granulomas with epithelioid cells, Langhans giant cells and a mononuclear infiltrate, with sparse or absent acid-fast bacilli. Mycobacterial culture is frequently negative, but tuberculin testing is positive. Lesions can be disfiguring and often persist for years where there is a risk of squamous metaplasia.

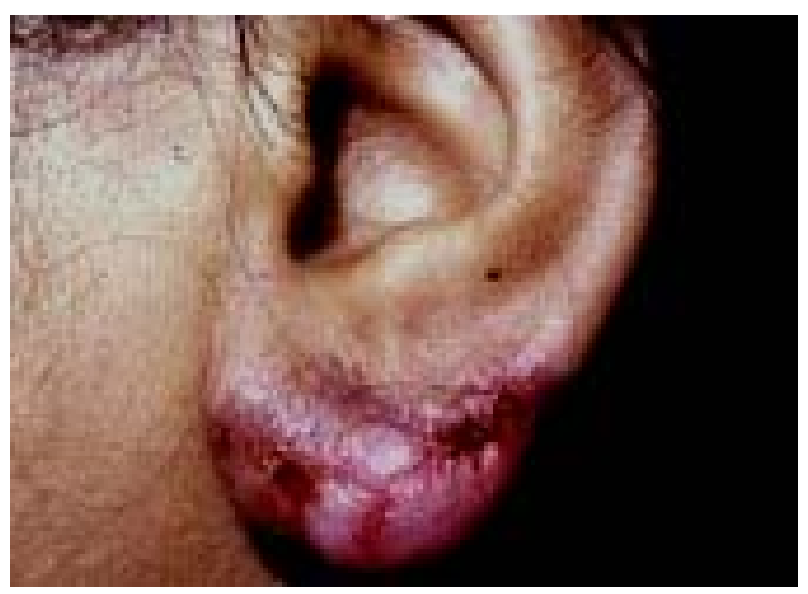

Figure 7. Lupus vulgaris.

\section{Tuberculids}

Tuberculids were once regarded as purely hypersensitivity reactions to the presence of mycobacteria in the host with an acquired immunity against TB. However, recent identification of mycobacterial DNA by polymerase chain reactions in affected tissue suggest that they are manifestations of hematogenous spread of bacilli in patients with tuberculin immunity, and therefore a form of true cutaneous $\mathrm{TB}^{15}$.

Typically, patients with tuberculids are in relatively good health and show the triad of positive tuberculin sensitivity, usually inactive tuberculous involvement of viscera or lymph nodes and negative staining and culture for pathogenic mycobacteria in affected tissue. Morphological variants of tuberculids are erythema induratum of Bazin, papulonecrotic tuberculid, Lichen scrofulosorum and other related conditions such as granulomatous mastitis and lupus miliaris disseminatus faciei.

Erythema induratum of Bazin is the most widely recognized tuberculid which occur predominantly in females. They occur as tender indurated plaques and nodules that may progress to ulceration and scarring at the posterior calves of legs. Later fat necrosis and foreign-body giant cells occur, and fibrosis and atrophy replace subcutaneous fat. This may occur with active or past disease and may recur for years ${ }^{16}$.

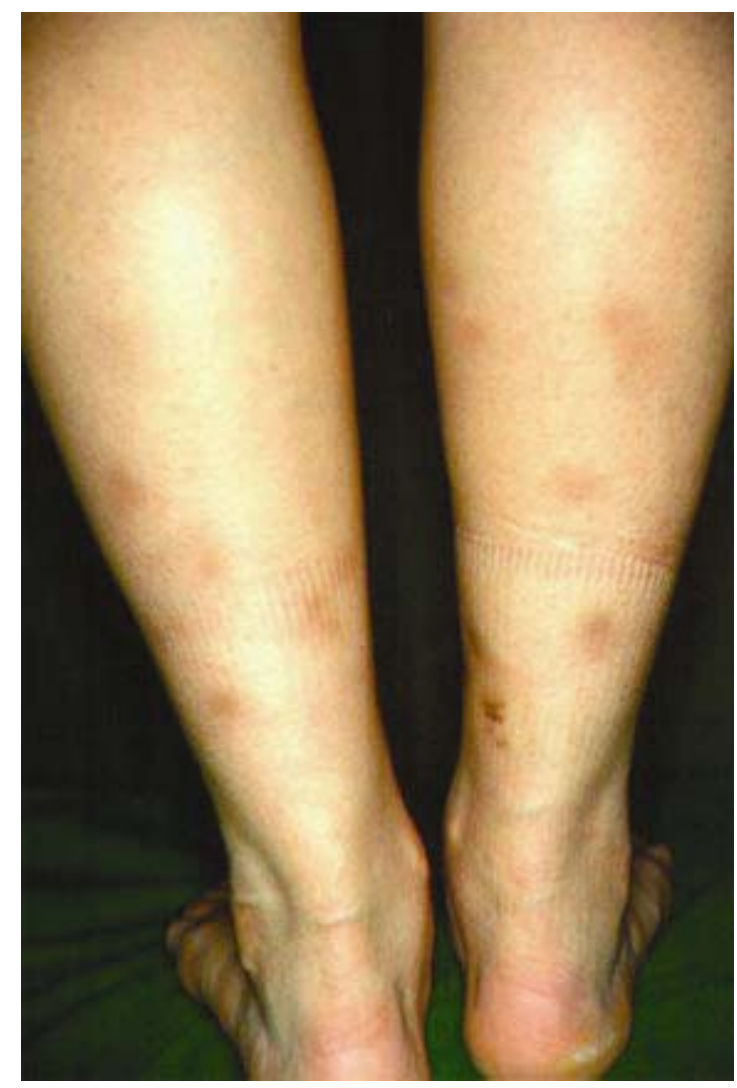

Figure 8. Erythema induratum of Bazin.

\section{Diagnosis and treatment}

A clinical diagnosis of skin tuberculosis should always be confirmed with biopsy in all cases and specimens should be stained and cultured for acidfast bacilli. A strongly positive Mantoux reaction of over $15 \mathrm{~mm}$ is considered of diagnostic value, while negative result does not exclude the diagnosis. Raised inflammatory markers would be supportive. If available, interferon gamma release assay (IGRA) and serological testing with ELISA or polymerase chain reaction would also be usefull ${ }^{17}$. However since too strict diagnostic microbiological criteria may result in under-diagnosis, therapeutic trials need to be considered in areas of high TB prevalence ${ }^{18}$.

As per the protocol per any case of extrapulmonary TB, all patients with skin TB should be thoroughly screened for associated pulmonary $T B$, with chest $X$ rays in all and sputum studies where relevant. Contact tracing is important in containing disease at a community level, especially in disease in children who are generally exposed to a small population only.

Management of skin TB depends on individual's previous TB status. Primary skin disease is considered less severe and category I regimen of anti-tubercular therapy (ATT) should be commenced. This comprises 
the standard six month regimen with a two-month intensive phase including isoniacid, rifampicin, pyrazinamide and ethambutol and a four-month continuation phase including isoniacid and rifampicin. In patients with past TB, category II regimen should be considered for retreatment of skin TB. This consists of a three-month intensive phase, where injectable streptomycin should be added for the first two months in addition to the standard four drugs. Continuation phase is also prolonged to five months. When treating children the dosage of medication should be calculated according to body weight and ethambutol preferably not given for the very young. A lower dosage regimen is considered for adults with a body weight below 30 kilograms and hepatic or renal disease, while a higher dose given for adults over 50 kilograms ${ }^{19,20,21}$.

Varying responses to ATT are shown by different types of skin TB. The commonest forms, lupus vulgaris and scrofuloderma generally show a good response to medical management. A clinical response is generally seen between 4 to 6 weeks of treatment, but a prolonged course is required for improvement of skin condition when present with coexisting miliary or disseminated disease, bone or joint disease or TB meningitis. Failure to respond to adequate therapy should raise the possibility of drug resistance, where the patient should be managed in a specialized centre with second line therapy ${ }^{22,23}$.

All patients on ATT should be frequently monitored for major and minor adverse effects of therapy, including impairment of colour vision, drug induced hepatitis or cholestasis and thrombocytopenia ${ }^{21,24}$. A bridging therapy is generally not required, if ATT needs to be withheld temporarily.
Surgical options such as electrosurgery, cryosurgery, and curettage with electro-desiccation are occasionally required for hypertrophic and verrucous forms of lupus vulgaris and TB verrucosa cutis. Reconstructive cosmetic surgery may be considered for disfiguring lesions.

\section{Discussion}

A high prevalence of extrapulmonary TB is an indicator of poor TB control in a community and early recognition, prompt treatment and effective contact tracing of all TB cases is mandatory to contain the disease. A thorough understanding of different presentations of TB is essential for all clinicians practicing in high prevalent settings, to achieve both national and global TB prevention targets.

Skin TB remains to be one of the most elusive and difficult diagnoses to make for dermatologists practicing in developing countries, not only because they have to consider a wider range of differential diagnoses such as leishmaniasis, leprosy, actinomycosis, skin cancer and deep fungal infections, but also because of the difficulty in obtaining a microbiological confirmation. Despite all the advances in microbiology, including sophisticated techniques such as polymerase chain reaction, the sensitivity of new methods are no better than the gold standard, that is, the isolation of organism in culture. Therefore even now we rely on diagnostic tools as old as the intradermal reaction to purified protein derivative (PPD) and therapeutic trials. These diagnostic difficulties may lead to serious case underreporting in low resource settings, which will obscure the true disease burden of the country.

Table 1. Classification of cutaneous tuberculosis

\begin{tabular}{|c|c|c|}
\hline Bacterial Load & Mechanism of Propagation & Disease form \\
\hline & (i) direct inoculation & Primary inoculation TB (chancre) \\
\hline \multirow[t]{3}{*}{ Multi-bacillary } & (ii) through contiguous infection & $\begin{array}{l}\text { Scrofuloderma } \\
\text { Tuberculosis periorificialis }\end{array}$ \\
\hline & (iii) hematogenous dissemination & $\begin{array}{l}\text { Acute miliary TB } \\
\text { Gumma (cold abscess) }\end{array}$ \\
\hline & (i) direct inoculation & $\begin{array}{l}\text { Tuberculosis verrucosa cutis } \\
\text { Lupus vulgaris (acral lesions) }\end{array}$ \\
\hline \multirow[t]{2}{*}{ Pauci-bacillary } & (ii) hematogenous dissemination & Lupus vulgaris (facial or multiple) \\
\hline & Tuberculids (immune mediated) & $\begin{array}{l}\text { Papulonecrotic tuberculid } \\
\text { Lichen scrofulosorum } \\
\text { Erythema induratum of bazin } \\
\text { Erythema nodosum }\end{array}$ \\
\hline
\end{tabular}




\section{References}

1. World health organization. Global tuberculosis control. WHO report 2010. (WHO/HTM/TB/2010.7)

2. World health organization. Trends in tuberculosis incidence and their determinants in 134 countries. WHO bulletin 2009; 87: 683-91.

3. Sharma SK, Mohan A. Extrapulmonary tuberculosis. Indian J Med Res 2004; 120: 316-53.

4. Bravo FG, Gotuzzo E. Cutaneous tuberculosis. Clinical Dermatology 2007; 25(2): 173-80.

5. Kumar B, Rai R, Kaur I, Sahoo B, Muralidhar S, Radotra BD. Childhood cutaneous tuberculosis: a study over 25 years from northern India. Int J Dermatol 2001; 40: 26-32.

6. Ho CK, Ho MH, Chong LY. Cutaneous tuberculosis in Hong Kong: an update. Hong Kong Med J 2006; 12 (4): 272-7.

7. Hamada M, Urabe K, Moroi Y, Miyazaki M, Furue M. Epidemiology of cutaneous tuberculosis in Japan: a retrospective study from 1906 to 2002. Int J Dermatol 2004; 43: $727-31$

8. Atukorala DN, Amarasekera LR. Tuberculosis of the skin in Sri Lanka. Cey Med J 1988; 33(3): 97-100.

9. Vyravanathan S, Nadarajah N. Tuberculosis of the skin over the sternum. Jaffna Medical Journal 1981; 16: 22-3.

10. De Silva HJ, Goonetilleke AK, De Silva NR, Amarasekera LR, Jayawickrama US. Erythema induratum (of Bazin) in a patient with endometrial tuberculosis. Postgrad Med J 1988; 64: 242-4.

11. Gawkrodger DJ. Mycobacterial infections. In: Champion RH, Burton JL. Ebling FJG, eds. Text Book of Dermatology 6th ed. London; Blackwell Scientific Publications 1998; 2: 1181-1214.

12. Tappeiner G, Wolff KD. Tuberculosis and other mycobacterial infections. In: Fitzpatrick TB, Eisen AZ, Wolff KD et al, eds. Dermatology in General Medicine 4th ed. McGraw Hill, Inc, New York 1993: 2370-94.

13. Beyt Jr BE, Ortbals DW, Santa Cruz DJ, Kobayashi GS Eisen AZ, et al. Cutaneous mycobacteriosis: analysis of 34 cases with a new classification of the disease. Medicine (Baltimore) 1981; 60: 95-109.
14. Tigoulet F, Fournier V, Caumes E. Clinical forms of the cutaneous tuberculosis. Bulletin Soc Pathol Exot 2003; 96: 362- 7.

15. Tan $\mathrm{SH}, \operatorname{Tan} \mathrm{BH}, \mathrm{Goh} \mathrm{CL}$, et al. Detection of Mycobacterium tuberculosis DNA using polymerase chain reaction in cutaneous tuberculosis and tuberculids. Int J Dermatol 1999; 38: 122-7.

16. Schneider JW, Jordaan HF, Geiger DH, et al. Erythema induratum of Bazin. A clinicopathological study of 20 cases and detection of Mycobacterium tuberculosis DNA in skin lesions by polymerase chain reaction. Am J Dermatopatho 1995; 17(4): 350-6.

17. Barbagallo $\mathrm{J}$, Tager $\mathrm{P}$, Ingleton $\mathrm{R}$, et al. Cutaneous tuberculosis: diagnosis and treatment. Am J Clin Dermatol 2002; 3(5): 319-28.

18. Sehgal V, Sardana K, Sehgal R, Sharma S. The use of antitubercular therapy as a diagnostic tool in pediatric cutaneous tuberculosis. Int J Dermatol 2005; 44: 1-3.

19. World health organization. Treatment of tuberculosis: guidelines (4th edition). WHO publication 2010. (WHO/HTM/ TB/2009.420)

20. Handog EB, Gabriel TG, Pineda RT. Management of cutaneous tuberculosis. Dermatol Ther 2008; 21(3): 154-61.

21. National programme for tuberculosis control and chest diseases. General manual for tuberculosis control. 2nd ed. Ministry of Health, Sri Lanka: 2005.

22. Ramam M, Mittal R, Ramesh V. How soon does cutaneous tuberculosis respond to treatment? Implications for a therapeutic test of diagnosis. Int J Dermatol 2005; 44(2): 121-4.

23. Nanda S, Rajpal M, Reddy BS. Multidrug-resistant cutaneous tuberculosis: response to therapy. Pediatr Dermatol 2003; 20: 545-7.

24. Yee D, Valiquette C, Pelletier M, Parisien I, Rocher I, Menzies D. Incidence of serious side effects from first-Line antituberculosis drugs among patients treated for active tuberculosis. Amer J Respi Crit Care Med 2003; 167: 1472-77. 